\title{
Impacto orçamentário do parto vaginal espontâneo e da cesariana eletiva sem indicação clínica no Brasil
}

\author{
Aline Piovezan Entringer, ${ }^{1}$ Maria Auxiliadora de Souza Mendes Gomes, ${ }^{1}$ \\ Ana Carolina Carioca da Costa $^{1}$ e Márcia Pinto ${ }^{1}$
}

Como citar Entringer AP, Gomes MASM, Costa ACC, Pinto M. Impacto orçamentário do parto vaginal espontâneo e da cesariana eletiva sem indicação clínica no Brasil. Rev Panam Salud Publica. 2018;42:e116. https:/ / doi.org/10.26633/RPSP.2018.116

RESUMO Objetivos. Estimar o impacto orçamentário do excesso de cesarianas sem indicação clínica em comparação ao parto vaginal para gestantes de risco habitual no Sistema Único de Saúde (SUS) no Brasil.

Métodos. A análise se baseou em um modelo estático. A população de referência foi a de gestantes de risco habitual. O horizonte temporal foi de 5 anos. Utilizou-se um modelo de regressão de Poisson para projetar o número de nascidos vivos de 2016 a 2020. O cálculo do custo direto da cesariana eletiva e do parto vaginal foi baseado em dois estudos prévios, nos quais foi calculado o valor esperado dos procedimentos através de um modelo de decisão analítico que incluiu as intercorrências clínicas da internação até a alta da maternidade. O cenário de referência dessa análise considerou $29 \%$ de cesarianas em excesso no país.

Resultados. O custo total da assistência ao parto e nascimento para as primíparas e multíparas sem cicatriz uterina no cenário de referência foi de US\$ 707,5 milhões para o ano de 2016. No cenário 1 (melhor cenário), que considerou apenas o parto vaginal para essas gestantes, houve uma redução de custos de US\$ 76,5 milhões ao ano. Para multíparas, a comparação do cenário de referência com o melhor cenário gerou economia de mais de US\$ 4 milhões ao ano. Conclusões. Os resultados indicam que o incentivo ao parto vaginal gera economia.

Palavras-chave

Cesárea; parto normal; gestão em saúde; avaliação em saúde; economia da saúde; Brasil.

Estimativas globais indicam que o custo das cesarianas desnecessárias em 137 países é de aproximadamente 2,32 bilhões de dólares (1). Diversos fatores influenciam as taxas de cesariana nos diversos países, como as características demográficas, clínicas e obstétricas, o modelo de

\footnotetext{
Fundação Oswaldo Cruz, Instituto Nacional de Saúde da Mulher, da Criança e do Adolescente Fernandes Figueira (IFF/FIOÇUZ), Rio de Janeiro (RJ), Brasil. Correspondência: Aline Piovezan Entringer, aline.entringer@iff.fiocruz.br
}

atenção obstétrica e a preferência dos profissionais de saúde e das mulheres. Quando realizada com justificativa clíni$\mathrm{ca}$, a cesariana diminui riscos maternos e neonatais e causa impacto positivo na morbimortalidade. No entanto, sem indicação clínica, pode estar relacionada ao aumento de desfechos negativos em saúde (2-6).

A taxa de cesariana é utilizada como marcador de qualidade da assistência à saúde, uma vez que seu aumento pode refletir um acompanhamento pré-natal inadequado ou indicações equivocadas do parto cesáreo em detrimento do parto vaginal (7). Conforme a Organização Mundial da Saúde (OMS), taxas de cesariana acima de $10 \%$ não estão relacionadas a redução da mortalidade materna e neonatal (8).

No Brasil, diretriz recente considera que a taxa de cesariana de referência, ajustada para a população brasileira, seria de $25 \%$ a $30 \%$ (9). Contudo, o Brasil 
está distante de alcançá-la: entre 2001 e 2014, as cesarianas cresceram $67 \%$ no país, tornando-se o método de nascimento prevalente, representando $57 \%$ dos nascimentos em 2014 (10). Agrega-se a esse contexto o fato de que o parto e a atenção obstétrica corresponderam a quase $20 \%$ do total das 11,4 milhões de internações realizadas no Sistema Único de Saúde (SUS), o que correspondeu a um gasto de $7 \%$ de todos os recursos gastos em internações (11).

Um estudo que calculou a razão de custo-efetividade incremental para o parto vaginal e a cesariana eletiva, sem indicação clínica, para gestantes primíparas de risco habitual em hospitais da região Sudeste do Brasil, identificou que o parto vaginal espontâneo é mais eficiente que a cesariana eletiva em termos de custos e geração de benefícios para as gestantes e os recém-nascidos $(12,13)$. Esses resultados, ainda que mostrem o custo de oportunidade, não permitem estimar o impacto orçamentário desses procedimentos. Sendo assim, o objetivo deste estudo foi estimar o impacto orçamentário das cesarianas sem indicação clínica em comparação ao parto vaginal para gestantes de risco habitual no SUS.

\section{MATERIAIS E MÉTODOS}

Trata-se de uma análise de impacto orçamentário baseada em um modelo estático que utilizou uma calculadora de custos desenvolvida em planilha eletrônica determinística elaborada em Excel e que incorporou, para o parto vaginal e o parto cesáreo, parâmetros populacionais e epidemiológicos (número de nascidos vivos, mulheres em idade fértil, taxa de cesariana e número de cesáreas prévias) e custo da assistência prestada para ambos os procedimentos. A perspectiva da análise é a do SUS, responsável pela prestação da assistência médica ao parto no Brasil. O horizonte temporal correspondeu ao período de 5 anos (2016 a 2020). O projeto que deu origem a este artigo foi aprovado pelos comitês de ética em pesquisa das instituições envolvidas (protocolos 44387715.1.0000.5269; 44387715.1.3002.5132 e 44387715.1. 3001.5279).

O parto vaginal espontâneo foi comparado com a cesariana eletiva, sem indicação clínica, através da intenção de tratar. O grupo "parto vaginal" correspondeu às gestantes que entraram em trabalho de parto espontaneamente, mas que poderiam ter sido submetidas a cesariana intraparto. O parto por via vaginal considerado foi o parto espontâneo ou com necessidade de assistência (fórcipe ou vácuo-extrator). O comparador foi o grupo "cesariana eletiva". O conceito de cesariana eletiva se aplica às gestantes em quem a intervenção cirúrgica foi programada e realizada antes do início do trabalho de parto, encontrando-se as membranas amnióticas íntegras. Neste estudo, considerou-se a cesariana eletiva sem justificativa clínica.

\section{População de referência}

A população de referência foi a de gestantes de risco habitual. Neste estudo, a gestante de risco habitual é aquela sem qualquer complicação clínica e obstétrica até o momento do parto, com feto único, a termo e apresentação cefálica, cujo nascimento poderia ocorrer tanto por parto vaginal quanto por cesariana. Como a recomendação atual para taxa de cesariana no Brasil é de $25 \%$ a $30 \%$ (9), adotamos como premissa a exclusão de $25 \%$ de gestantes da nossa análise para todos os cenários, uma vez que essa população iria se beneficiar do parto cirúrgico. As demais gestantes seriam de risco habitual e poderiam ser submetidas a ambos os procedimentos. O cenário de referência considerou que as cesarianas em excesso seriam aquelas que ultrapassassem os $25 \%$ que foram excluídos.

\section{Análise dos dados}

O acesso da população à atenção à saúde ocorre no país por meio do SUS, de acesso universal e financiado exclusivamente com recursos públicos, e através da saúde suplementar, que corresponde ao segmento de planos privados de saúde, de vinculação eletiva, financiado com recursos das famílias e/ou dos empregadores e mediante o desembolso direto (14). A saúde suplementar no Brasil é regulada pela Agência Nacional de Saúde Suplementar.

A análise estatística aplicou um modelo de regressão de Poisson para estimar o número de partos vaginais e cesáreos anualmente de 2016 a 2020 para a população brasileira de mulheres em idade fértil como offset (15) a partir de dois cenários: sistema de saúde suplementar e SUS. As estimativas populacionais foram obtidas a partir dos dados de censos e contagens populacionais (16). A série histórica de nascidos vivos em que se baseou o modelo estatístico foi obtida através do Sistema de Informações sobre Nascidos Vivos (SINASC) (10).

As informações do SINASC correspondem a todos os nascidos vivos do Brasil, independentemente do tipo de prestador. Para utilizar os dados do SINASC, obtivemos informações de nascidos vivos por tipo de parto da Agência Nacional de Saúde Suplementar e excluímos essa população da análise, permanecendo apenas com os nascidos vivos do SUS (figura 1).

\section{Custos}

Os dados para o cálculo do custo direto da cesariana eletiva e parto vaginal basearam-se em dois estudos de custo e de custo-efetividade realizados a priori $(12,13)$. Foi calculado o custo direto dos procedimentos, da assistência ao recém-nascido em sala de parto, da permanência em alojamento conjunto para mãe, recém-nascido e acompanhante, das intercorrências maternas clínicas (hemorragia com necessidade de transfusão e trombose/embolia) e cirúrgicas (histerectomia e ruptura uterina) e da diária em unidade de terapia intensiva (UTI)/ adulto (mãe) e neonatal. Foram incluídas consultas com médicos e enfermeiros, medicamentos, materiais hospitalares, custos administrativos e depreciação de equipamentos através da técnica mista de microcusteio e topdown. A partir de um modelo de decisão analítico (árvore de decisão), foi estimado o valor esperado do parto vaginal e da cesariana eletiva a partir da utilização dos recursos de saúde e das probabilidades de intercorrências clínicas e cirúrgicas para a mãe e o recém-nascido no período entre a permanência hospitalar até a alta, incluindo a probabilidade cesariana intraparto para o grupo parto vaginal $(12,13)$. $\mathrm{O}$ valor esperado obtido do modelo de decisão, que incluiu custos e resultados em saúde, foi considerado para o cálculo do impacto orçamentário (tabela 1).

Todos os custos foram convertidos em dólares dos Estados Unidos usando uma taxa de conversão de US\$1,00 = R \$ 3,18, que foi a média de janeiro a agosto de 2017.

\section{Análise de sensibilidade}

Construímos três cenários alternativos ao caso-base com o objetivo de 
FIGURA 1. Projeção populacional de partos vaginais e cesáreos por tipo de prestador, Brasil, 2016 a 2020
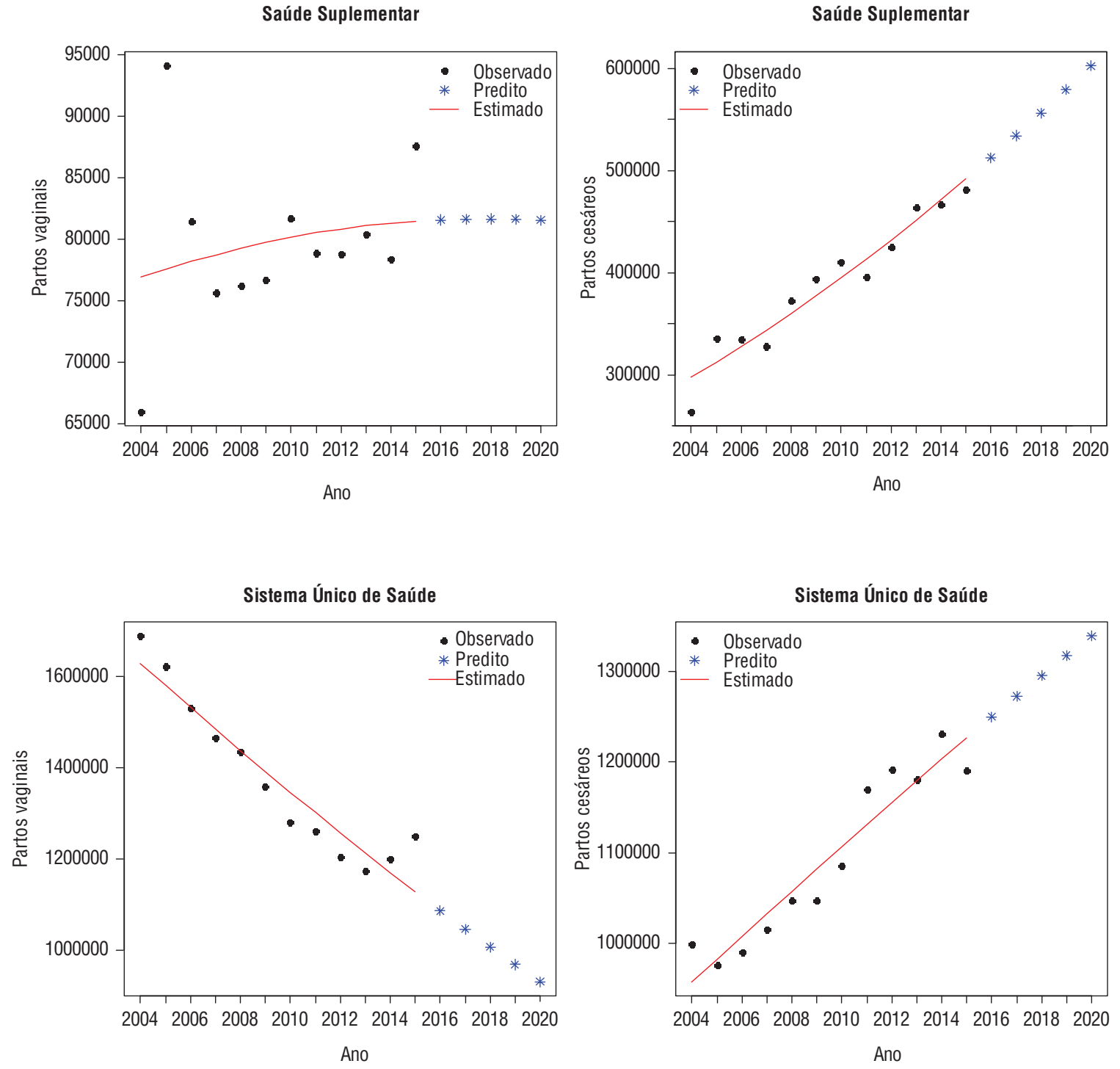

TABELA 1. Variáveis do modelo de impacto orçamentário da realização de partos vaginais e cesáreos, Brasil, 2016 a 2020

\begin{tabular}{lcc}
\hline Variável & Parâmetro & Referência \\
\hline Percentual de gestantes sem cesariana prévia (primíparas e multíparas) & $67 \%$ & 4 \\
Percentual de multíparas com uma cesariana prévia & $19 \%$ & 4 \\
Percentual de cesarianas esperadas/necessárias & $25 \%$ & 3 \\
Valor esperado do trabalho de parto & & \\
$\quad$ Primíparas e multíparas sem cicatriz & US\$537,60 & 13 \\
$\quad$ Multíparas com uma cicatriz & US $\$ 621,12$ & 13 \\
Valor esperado da cesariana eletiva & & \\
$\quad$ Primíparas e multíparas sem cicatriz & US\$ 706,25 & 13 \\
$\quad$ Multíparas com uma cicatriz & US $\$ 652,31$ & 13 \\
\hline
\end{tabular}

simular a redução e o aumento da taxa de cesariana ao longo do horizonte analítico de 5 anos. O cenário 1 (melhor cenário) representa a atual recomendação das diretrizes brasileiras $(25 \%$ de cesarianas para a população) (9). Como $25 \%$ das gestantes são submetidas à cesariana necessária (excluídas da análise), seriam esperados $75 \%$ de partos vaginais para as demais gestantes, consideradas neste estudo como de risco habitual. O cenário 2 (intermediário) pressupõe uma redução gradual de 3 pontos percentuais ao ano na taxa de cesariana. $\mathrm{O}$ cenário 3 (pior cenário) considerou um crescimento de 1 ponto percentual ao ano na taxa de cesariana.

\section{Cálculo do impacto orçamentário}

A população de referência foi subdividida em dois grupos: gestantes de risco habitual, primíparas ou multíparas, sem cicatriz uterina prévia; e gestantes de risco habitual, multíparas, com uma cicatriz uterina prévia. Gestantes com duas ou mais cesarianas prévias foram excluídas. 
O percentual de primíparas e multíparas foi obtido a partir da base de dados completa do SINASC de 2014. Foi utilizada a variável "número de cesarianas" para se obter o percentual de mulheres sem cesariana e com uma cesariana prévia (17) (tabela 1).

O custo total foi calculado multiplicando-se a população elegível pelo valor esperado do modelo de decisão analítico $(12,13)$, que considerou custos dos procedimentos e intercorrências clínicas (tabela 1). O impacto orçamentário foi calculado a partir da subtração do custo total da assistência de cada um dos três cenários pelo cenário de referência. Caso o resultado da subtração fosse positivo, indicaria um aumento de custo para o SUS; caso fosse negativo, o resultado indicaria economia.

\section{RESULTADOS}

O modelo foi revisado e testado após a sua conclusão a fim de identificar possíveis erros relacionados com a incorporação dos dados e a sintaxe da programação das variáveis. Essa validação interna garantiu que os cálculos matemáticos estivessem exatos e consistentes com todos os parâmetros e pressupostos estabelecidos pelo modelo. Os resultados dessa análise de impacto orçamentário são apresentados para as duas populações separadamente, a partir do caso-base e dos três cenários propostos.

\section{Análise de impacto orçamentário para gestantes de risco habitual primíparas e multíparas sem cicatriz uterina}

O custo total da assistência ao parto e nascimento para as mulheres de risco habitual primíparas e multíparas sem cicatriz prévia no SUS para o caso base foi de US\$707,5 milhões para o ano de 2016, considerando a população elegível (cerca de $40 \%$ de todos nascimentos no ano) (tabela 2). Apesar do aumento da taxa de cesariana ao longo dos anos, a tendência é de redução nos custos, uma vez que há queda no número de nascidos vivos. Ao ser comparado com cenário de referência, o melhor cenário, que considerou apenas o parto vaginal para gestantes de risco habitual, geraria uma economia de quase US\$ 76,5 milhões por ano, ou seja, mais de US\$ 400 milhões ao longo dos 5 anos avaliados.

O cenário intermediário (redução de 3 pontos percentuais ao ano na taxa de cesariana) poderia gerar uma economia de US\$ 39 milhões em 5 anos. O pior cenário mostrou um aumento gradual nas taxas de cesariana e aumentaria o impacto orçamentário em cerca de US\$ 13 milhões nos 5 anos. Nesse cenário, o aumento de 1 ponto percentual na taxa de cesariana em cima do cenário de referência acarretaria um acréscimo de US\$2,5 milhões ao ano.

\section{Análise de impacto orçamentário da cesariana em gestantes multíparas de risco habitual com cicatriz uterina prévia}

As gestantes de risco habitual, multíparas com cicatriz uterina prévia, atendidas no SUS, corresponderam a cerca de $11,4 \%$ dos nascimentos ao ano. O custo da atenção ao parto e nascimento para essa população foi de US\$ 210 milhões para o cenário de referência em 2016. Nos anos subsequentes houve uma redução nos

TABELA 2. Impacto orçamentário do parto cesáreo em gestantes de risco habitual, primíparas e multíparas sem cicatriz uterina prévia no Sistema Único de Saúde, Brasil, 2016 a 2020

\begin{tabular}{|c|c|c|c|c|c|}
\hline Impacto/cenário & 2016 & 2017 & 2018 & 2019 & 2020 \\
\hline \multicolumn{6}{|l|}{ Impacto orçamentário } \\
\hline Nascidos vivos & 2929827 & 2933931 & 2939212 & 2945642 & 2953175 \\
\hline Nascidos vivos no SUS & 2335827 & 2318280 & 2301359 & 2285039 & 2269279 \\
\hline Nascidos vivos no SUS (gestantes sem cicatriz uterina) & 1565004 & 1553248 & 1541911 & 1530976 & 1520417 \\
\hline$\%$ de cesarianas no SUS & 54 & 55 & 56 & 58 & 59 \\
\hline \multicolumn{6}{|l|}{ Cenário de referência } \\
\hline$\%$ de cesarianas em excesso SUS & 29 & 30 & 31 & 33 & 34 \\
\hline$\%$ de parto vaginal no SUS & 46 & 45 & 44 & 42 & 41 \\
\hline Custo total da assistência (US\$) & 707552227,43 & 704856434,24 & 702312026,54 & 702495329,97 & 700214247,30 \\
\hline \multicolumn{6}{|l|}{ Cenário 1 (melhor cenário) } \\
\hline$\%$ de cesarianas em excesso SUS & $0 \%$ & $0 \%$ & $0 \%$ & $0 \%$ & $0 \%$ \\
\hline$\%$ de parto vaginal no SUS & $75 \%$ & $75 \%$ & $75 \%$ & $75 \%$ & $75 \%$ \\
\hline Custo total da assistência (US\$) & 631014078,34 & 626273828,30 & 621702689,59 & 617293908,57 & 613036409,24 \\
\hline Impacto orçamentário (US\$) (cenário 1 - referência) & $-76538149,08$ & $-78582605,94$ & $-80609336,95$ & $-85201421,41$ & $-87177838,06$ \\
\hline \multicolumn{6}{|l|}{ Cenário 2 (cenário intermediário) } \\
\hline \% de cesarianas em excesso SUS & 26 & 27 & 28 & 30 & 31 \\
\hline$\%$ de parto vaginal no SUS & 49 & 48 & 47 & 45 & 44 \\
\hline Custo total da assistência (US\$) & 699634487,87 & 696998173,65 & 694511122,97 & 694749746,21 & 692522085,12 \\
\hline Impacto orçamentário (US\$) (cenário 2 - referência) & $-7917739,56$ & $-7858260,59$ & $-7800903,58$ & $-7745583,76$ & $-7692162,18$ \\
\hline \multicolumn{6}{|l|}{ Cenário 3 (pior cenário) } \\
\hline \% de cesarianas em excesso SUS & 30 & 31 & 32 & 34 & 35 \\
\hline$\%$ de parto vaginal no SUS & 45 & 44 & 43 & 41 & 40 \\
\hline Custo total da assistência (US\$) & 710191473,95 & 707475854,44 & 704912327,73 & 705077191,23 & 702778301,36 \\
\hline Impacto orçamentário (US\$) (cenário 3 - referência) & 2639246,52 & 2619420,20 & 2600301,19 & 2581861,25 & 2564054,06 \\
\hline
\end{tabular}


custos devido à queda no número de nascidos vivos. $\mathrm{O}$ melhor cenário mostrou que, com realização de parto vaginal para todas essas gestantes, a economia seria de quase US\$21,5 milhões ao longo de 5 anos. A redução gradual da cesariana (cenário 2) nesse período geraria uma economia de US\$ 2 milhões. No entanto, com o crescimento da taxa de cesarianas em 1 ponto percentual ao ano, haveria um aumento de cerca de US\$ 680 mil nos 5 anos em relação ao cenário de referência (tabela 3).

\section{DISCUSSÃO}

A comparação entre o cenário de referência e o melhor cenário mostrou que a taxa de cesarianas em excesso projetada de 2016 a 2020 geraria um impacto de mais de US\$ 80 milhões ao ano para o SUS, considerando as gestantes primíparas e multíparas incluídas no estudo. $\mathrm{O}$ crescimento gradual dessa taxa em apenas 1 ponto percentual ao ano (cenário 3) aumentaria esse valor em quase US\$ 3 milhões ao ano.

Não foram encontrados outros estudos que calculassem impacto orçamentário desses procedimentos para o SUS. Estudo multicêntrico em 137 países, realizado pela OMS, calculou o número de cesáreas necessárias e estimou os custos envolvidos nas categorias de cesáreas "necessárias" e "em excesso". O estudo identificou que 3,2 milhões de cesáreas são necessárias, o que corresponde a um custo de US\$ 432 milhões, e que 6,2 milhões de cesáreas estão em excesso (50\% dessas na China e no Brasil), correspondendo a um custo cinco vezes maior (US\$2,32 bilhões) (1). No entanto, esse estudo da OMS não realizou comparações com o custo do parto normal, utilizou critérios de definição de "cesarianas em excesso" diferentes dos utilizados no presente estudo e não apresentou resultados separados para cada população (com e sem cicatriz uterina) e tipo de prestador.

A análise de custo-efetividade que embasou este estudo identificou que o parto vaginal é mais eficiente que a cesariana eletiva para primíparas, mas não apresentou resultados robustos para as multíparas com uma cicatriz uterina prévia (13). No entanto, optamos por incluir as gestantes multíparas com uma cicatriz prévia nesta análise de impacto orçamentário, uma vez que a recomendação do Ministério da Saúde do Brasil para essas gestantes indica que o parto vaginal pode ser realizado na maioria das situações e deve ser realizado sempre em ambiente hospitalar, após aconselhamento adequado e assinatura do termo de consentimento (9).

Uma das limitações deste estudo se refere à utilização de um modelo de regressão de Poisson para obter estimativas de população para os anos de 2016 a 2020, uma vez que essa informação não estava disponível. Outra limitação foi a utilização do percentual de referência de cesarianas para o Brasil (25\%) para excluir gestantes que poderiam se beneficiar da cesariana, sendo que as demais gestantes foram consideradas como de risco habitual. Diferentes taxas de cesariana encontradas entre os países, as regiões e entre prestadores têm desencadeado intenso debate acerca do percentual ideal dos partos cirúrgicos, suas indicações e as consequências da cesariana eletiva para a saúde da mãe e do bebê. A recomendação quanto às taxas de cesariana no Brasil (25 a 30\%) é dinâmica - ou seja, a partir do momento em que houver redução na

TABELA 3. Impacto orçamentário do parto cesáreo em gestantes de risco habitual multíparas com 1 cicatriz uterina prévia no Sistema Único de Saúde, Brasil, 2016 a 2020

\begin{tabular}{|c|c|c|c|c|c|}
\hline Impacto/cenário & 2016 & 2017 & 2018 & 2019 & 2020 \\
\hline \multicolumn{6}{|l|}{ Impacto orçamentário } \\
\hline Nascidos vivos & 2929827 & 2933931 & 2939212 & 2945642 & 2953175 \\
\hline Nascidos vivos no SUS & 2335827 & 2318280 & 2301359 & 2285039 & 2269279 \\
\hline Nascidos vivos no SUS (gestantes com 1 cicatriz) & 443807 & 440473 & 437258 & 434157 & 431163 \\
\hline$\%$ de cesarianas no SUS & 54 & 55 & 56 & 58 & 59 \\
\hline \multicolumn{6}{|l|}{ Cenário de referência } \\
\hline$\%$ de cesarianas em excesso SUS & 29 & 30 & 31 & 33 & 34 \\
\hline$\%$ de parto vaginal no SUS & 46 & 45 & 44 & 42 & 41 \\
\hline Custo total da assistência (US\$) & 210756651,12 & 209310786,94 & 207919400,16 & 206715736,25 & 205424470,52 \\
\hline \multicolumn{6}{|l|}{ Cenário 1 (melhor cenário) } \\
\hline$\%$ de cesarianas em excesso SUS & 0 & 0 & 0 & 0 & 0 \\
\hline$\%$ de parto vaginal no SUS & 75 & 75 & 75 & 75 & 75 \\
\hline Custo total da assistência (US\$) & 206742945,96 & 205189869,27 & 203692199,54 & 202247724,04 & 200852813,88 \\
\hline Impacto orçamentário (US\$) (cenário 1 - referência) & $-4013705,16$ & $-4120917,66$ & $-4227200,62$ & $-4468012,21$ & $-4571656,65$ \\
\hline \multicolumn{6}{|l|}{ Cenário 2 (cenário intermediário) } \\
\hline \% de cesarianas em excesso SUS & 26 & 27 & 28 & 30 & 31 \\
\hline$\%$ de parto vaginal no SUS & 49 & 48 & 47 & 45 & 44 \\
\hline Custo total da assistência (US\$) & 210341440,24 & 208898695,17 & 207510316,23 & 206309553,32 & 205021089,05 \\
\hline Impacto orçamentário (US\$) (cenário 2 - referência) & $-415210,88$ & $-412091,77$ & $-409083,93$ & $-406182,93$ & $-403381,47$ \\
\hline \multicolumn{6}{|l|}{ Cenário 3 (pior cenário) } \\
\hline \% de cesarianas em excesso SUS & 30 & 31 & 32 & 34 & 35 \\
\hline$\%$ de parto vaginal no SUS & 45 & 44 & 43 & 41 & 40 \\
\hline Custo total da assistência (US\$) & 210895054,75 & 209448150,86 & 208055761,47 & 206851130,56 & 205558931,01 \\
\hline Impacto orçamentário (US\$) (cenário 3 - referência) & 138403,63 & 137363,92 & 136361,31 & 135394,31 & 134460,49 \\
\hline
\end{tabular}


taxa atual de cesariana e, consequentemente, redução no número de mulheres com cesarianas prévias, essa referência sugerida pela diretriz brasileira pode ser alterada. Ademais, as projeções até 2020 foram baseadas em uma série histórica ampla, porém o número de nascidos vivos pode ser alterado de acordo com eventos epidemiológicos que podem ocorrer no Brasil e afetar a população de mulheres em idade fértil.

A tendência atual no Brasil ainda é de crescimento nas taxas de cesariana e, segundo a projeção aqui realizada, se essa tendência de crescimento dos últimos 15 anos (2000 a 2014) se mantiver, a taxa de partos cirúrgicos pode alcançar quase $60 \%$ no SUS e quase $90 \%$ na saúde suplementar até 2020. Sabemos que a queda nas taxas de cesarianas não ocorrerá de forma abrupta, mas AP, Merialdi M, Althabe F. The global numbers and costs of additionally needed and unnecessary caesarean sections performed per year: overuse as a barrier to universal coverage. World Health Report 2010. (Background Paper No. 30). Genebra: WHO: 2010. Disponível em: http://www. who.int/healthsystems / topics / financing/healthreport/30C-sectioncosts.pdf Acessado em junho de 2018.

2. Geller EJ, Wu JM, Jannelli ML, Nguyen TV, Visco AG. Neonatal outcomes associated with planned vaginal versus planned primary cesarean delivery. J Perinatol. 2010; 30(4):258-64.

3. Geller EJ, Wu JM, Jannelli ML, Nguyen TV, Visco AG. Maternal outcomes associated with planned vaginal versus planned primary cesarean delivery. Am J Perinatol. 2010;27(9):675-83.

4. Liu S, Liston RM, Joseph KS, Heaman M, Sauve R, Kramer MS, et al. Maternal mortality and severe morbidity associated with low-risk planned cesarean delivery versus planned vaginal delivery at term. CMAJ. 2007;176(4):455-60.

5. MacDorman MF, Declercq E, Menacker F, Malloy MH. Neonatal mortality for primary cesarean and vaginal births to low-risk women: application of an "intention-totreat" model. Birth. 2008;35(1):3-8.

6. Allen V, O'Connell C, Liston R, Baskett T. Maternal morbidity associated with cesarean delivery without labor compared with spontaneous onset of labor at term. Obstet Gynecol. 2003;102(3):477-82.

7. Agência Nacional de Saúde Suplementar. Taxa de parto cesáreo. Disponível em: http://bvsms.saude.gov.br/bvs/publicacoes/qualificacao_saude_sup/pdf/

gradualmente, a partir de políticas públicas que incentivem práticas menos invasivas para a atenção do parto e nascimento, uso restrito da episiotomia, práticas que aumentem a satisfação da mulher, auditoria e controle das indicações de cesarianas, apoio dos centros de parto intra-hospitalares e inserção da enfermagem obstétrica nas equipes de plantão, considerando o impacto positivo de sua atuação na qualidade da atenção (18).

Os arranjos institucionais e assistenciais que se observam atualmente na organização da atenção obstétrica no Brasil indicam que a tomada de decisão tendo como referência o gerenciamento de recursos de saúde, a eficácia e a efetividade deve ser considerada, mas não é suficiente. Este estudo pretendeu contribuir para a gestão da atenção obstétrica e perinatal

\section{REFERÊNCIAS}

Atenc saude2fase.pdf Acessado em junho de 2018.

8. Organização Mundial da Saúde (OMS). Declaração da OMS sobre taxas de cesáreas. Genebra: OMS; 2015. Disponível em: http:/ / apps.who.int/iris/bitstream/10665/ 161442/3/WHO_RHR_15.02_por.pdf Acessado em outubro de 2016.

9. Brasil, Ministério da Saúde. Diretrizes de atenção à gestante: a operação cesariana. Brasília: Comissão Nacional de Incorporação de Tecnologias no SUS; 2016. Disponível em: http://conitec.gov.br/images/ Consultas/Relatorios/2016/Relatorio Diretrizes_Cesariana_N179.pdf Acessado em junho de 2018.

10. Brasil, Ministério da Saúde. Sistema de Informações sobre Nascidos Vivos SINASC. DATASUS. Disponível em: http://tabnet.datasus.gov.br/cgi/tabcgi. exe?sinasc/cnv/nvuf.def Acessado em junho de 2018.

11. Brasil, Ministério da Saúde. Internações hospitalares no SUS - por local de internação - Brasil. DATASUS. Disponível em: http://tabnet.datasus.gov.br/cgi/deftohtm.exe?sih/cnv/sxuf.def Acessado em fevereiro de 2014

12. Entringer AP, Pinto MT, Gomes MASM. Análise de custos da atenção hospitalar ao parto vaginal e à cesariana eletiva para gestantes de risco habitual no Sistema Único de Saúde. Cienc Saude Colet. 2017/set.

13. Entringer AP, Pinto MT, Dias M, Gomes MASM. Análise de custo-efetividade do parto vaginal espontâneo e da cesariana eletiva para gestantes de risco habitual no Sistema Único de Saúde. Cad Saude Publica. 2018;34(5):e00022517.

14. Silveira DP, Araujo DV, Gomes GHG, Lima $\mathrm{KC}$, Estrella K, Oliveira M, et al. Idoso na e para mostrar que o incentivo ao parto vaginal, além de ser condizente com a atual política pública brasileira e internacional, geraria economia para o SUS. Apesar das limitações apontadas, nossa intenção foi a de apresentar o impacto orçamentário do parto vaginal e da cesariana eletiva a partir de cenários hipotéticos, mas que podem apoiar os gestores brasileiros no planejamento e gestão das políticas de saúde.

Conflitos de interesse. Nada declarado pelos autores.

Declaração. As opiniões expressas no manuscrito são de responsabilidade exclusiva dos autores e não refletem necessariamente a opinião ou políticas da RPSP/PAJPH ou da Organização Pan-Americana da Saúde (OPAS). saúde suplementar: uma urgência para a saúde da sociedade e sustentabilidade do setor. Rio de Janeiro: ANS; 2016. Disponível em: http://www.ans.gov.br/images/stories/Materiais_para_pesquisa/Materiais_ por_assunto/web_final_livro_idosos.pdf Acessado em junho de 2018.

15. Dobson AJ. An introduction to generalized linear models. $2^{a}$ ed. Chapman \& Hall/ CRC; Nova Iorque: 2002.

16. Instituto Brasileiro de Geografia e Estatística (IBGE). Censo demográfico 2010: característica da população e dos domicílios. Resultados do universo. Disponível em: http://www.ibge.gov.br/home/estatisti$\mathrm{ca} /$ populacao/censo2010/caracteristicas_ da_populacao/resultados_do_universo. pdf Acessado em junho de 2018.

17. Brasil, Ministério da Saúde, Departamento de Informática do SUS. Serviços. Transferência de arquivos. Arquivos de dados. Sistema de Informação de Nascidos Vivos. Disponível em: http://www2.datasus. gov.br/DATASUS /index.php? area $=0901$ \&item $=1$ \&acao $=28 \&$ pad $=31655$ Acessado em junho de 2018.

18. Johantgen M, Fountain L, Zangaro G, Newhouse R, Stanik-Hutt J, White K. Comparison of labor and delivery care provided by certified nurse-midwives and physicians: A systematic review, 1990 to 2008. Womens Health Issues. 2012;22(1): e73-81.

Manuscrito recebido em 19 de outubro de 2017 Aceito em versão revisada em 6 de maio de 2018 . 
ABSTRACT

Budgetary impact of spontaneous vaginal delivery and elective cesarean section without clinical indication in Brazil
Objective. To estimate the budgetary impact of excess cesarean deliveries without clinical indication compared to vaginal deliveries in the Unified Health System (SUS) in Brazil.

Methods. The analysis was based on a static model. The reference population was that of pregnant women at normal risk. The time horizon was 5 years. A Poisson regression model was used to estimate the number of live births from 2016 to 2020 . Calculation of the direct cost of elective cesarean delivery and vaginal delivery was based on two previous studies, in which the expected monetary value of the procedures was calculated through an analytical decision model that included all clinical events from admission until discharge. The reference scenario for this analysis considered $29 \%$ of excess cesareans in the country.

Results. The total cost of delivery and birth care for primiparous and multiparous women without uterine scar in the reference scenario was US\$ 707500000 for the year 2016. In scenario 1 (best scenario), which considered only vaginal delivery for these pregnant women, there was a cost reduction of US\$ 76500000 per year. For multiparous women, comparison of the reference scenario with the best scenario showed savings of more than US\$ 4000000 per year.

Conclusions. The results indicate that the incentive to vaginal delivery generates savings.

Keywords Cesarean section; natural childbirth; health management; health evaluation; health economics; Brazil.
RESUMEN

Impacto presupuestario del parto vaginal espontáneo y de la cesárea electiva sin indicación clínica en Brasil

Palabras clave
Objetivos. Estimar el impacto presupuestario del exceso de cesáreas sin indicación clínica en comparación con el parto vaginal para embarazadas con riesgo habitual en el Sistema Único de Salud (SUS) en Brasil.

Métodos. El análisis se basó en un modelo estático. Se empleó como referencia la población de embarazadas con riesgo habitual. El horizonte temporal fue de 5 años. Se utilizó un modelo de regresión de Poisson para proyectar el número de nacidos vivos del 2016 al 2020. El cálculo del costo directo de la cesárea electiva y del parto vaginal se basó en dos estudios previos, en los cuales se estimó el valor previsto de los procedimientos por medio de un modelo analítico de decisión que incluyó las complicaciones clínicas de la hospitalización hasta el alta del servicio de maternidad. En el escenario de referencia de ese análisis se consideró que en el país se realizan $29 \%$ de cesáreas en exceso.

Resultados. El costo total de la asistencia al parto y al nacimiento para las primíparas y multíparas sin cicatriz uterina en el escenario de referencia fue de US\$707 500000 en el año 2016. En el primer escenario (el mejor), en el cual se analizó solo el parto vaginal de esas embarazadas, hubo una reducción de costos de US\$76 500000 al año. Para multíparas, la comparación del escenario de referencia con el mejor generó ahorros superiores a US\$ 4000000 al año.

Conclusiones. Los resultados indican que el incentivo del parto vaginal genera ahorros.

Cesárea; parto normal; gestión en salud; evaluación en salud; economía de la salud; Brasil. 